\title{
State equation of mineral sands
}

\author{
Tomislav Ivšić • Astrid Gojmerac Ivšić
}

Received: 16 March 2010 / Published online: 23 December 2011

(C) The Author(s) 2011. This article is published with open access at Springerlink.com

\begin{abstract}
The pressure-volume analogy between compressible fluids and macroscopic sand bodies (Ivsic et al. in Phys A, 277:47-61, 2000) is further extended using quantitative determination of corresponding empirical constants based on adapted van der Waals state equation. The isothermal constants obtained by interpretation of triaxial sand tests at so called "critical state of sand" are clearly related to the universal ideal gas properties and molar properties of mineral sands. The corresponding constants for sand and gases or other volatile liquids have the same order of magnitude. The apparent bulk repulsion/attraction effects in sand bodies are also discussed.
\end{abstract}

Keywords Mineral sand · Van der Waals fluid - Critical state $\cdot$ Triaxial test $\cdot$ Molar properties

\section{Introduction}

Granular materials demonstrate rich phenomenology that in many aspects resembles that of the ordinary solids, liquids and gases. However, attempts to describe their behavior using standard concepts yield only partial success. Very often the

Electronic supplementary material The online version of this article (doi:10.1007/s10035-011-0301-4) contains supplementary material, which is available to authorized users.

T. Ivšić ( $\square)$

Geotechnical Department, Faculty of Civil Engineering, University of Zagreb, Kačićeva 26, 10000 Zagreb, Croatia

e-mail: tom@grad.hr

\section{A. G. Ivšić}

Department of Chemistry, Faculty of Science, University of Zagreb, Horvatovac 102a, 10000 Zagreb, Croatia

e-mail: agi@chem.pmf.hr behavior of granular materials is significantly different from what is expected in ordinary concepts, leading to "unusual" solids or fluids [1,2]. In attempt to explain this unique behavior, a number of approaches appeared, introducing various concepts on a large scale, from microscopic and mezoscopic level (grain to grain interactions or cluster behavior) to macroscopic level, where bulk properties of material are considered [3].

The level of understanding of even basic phenomena is not well correlated to the extent of research efforts, and it seems that the granular material behavior is still a challenging task for many researchers $[4,5]$.

In last few decades, an enormous number of papers related to granular material behavior have been published. Most of basic results or theories are compiled in review papers or books [1-3,6-8]. Many published studies often focus on particular concepts or explanations. Differences are made between static regime and dynamic regime involving granular flow [9-11]. In static configuration, where the grains are jammed, the problems of fabric, packing or force transmission are dominant. For granular flow two regimes are often distinguished: the slow, dense flow where the frictional component is dominant and the rapid flow regime where viscous effects can be found.

The granular materials physics is studied experimentally and theoretically. In the theoretical approach two distinct, but related methods, are usually used: statistical theories e.g. [12-16] based on particle dynamics and modified forms of kinetic gas theory, or the continuum mechanics-based theories introducing constitutive equations and stress tensor e.g. [17-19]. The thermodynamics of granular assemblies is also studied e.g. [20-23], or the principles of thermomechanics are applied e.g. [24-26].

The theoretical approach includes many idealizations and assumptions which go beyond the original theories. In 
numerical simulations (molecular dynamics or discrete element method) the discrete hard spheres concept is often applied. Also, the concepts of "granular temperature", or other analogues to ordinary temperature, are used to deal with thermodynamic variables like entropy.

In the presented work, a more classical, phenomenological approach is applied, using experiments on macroscopic sand bodies. These macroscopic systems involve bulk properties, where continuum idealization can be applied. The pressurevolume behavior of mineral sands is examined using the concept of compressible fluid described earlier by Ivsic, Galovic and Kirin [27] and summarized later in text.

The research in this paper is focused on the properties of the analogous fluid. The particular state examined here is so called "critical state of sand" (CSS) where the yielding or failure of sand body takes place. The CSS was obtained experimentally and also confirmed by recent numerical simulations [28-33]. It can be characterized as a quasi-stationery state where the thermodynamic potentials, pressure and volume of the body, reach constant values in isothermal conditions.

In terms of novel granular literature, this state of yielding sand can be recognized as being near (or at) the state of jamming transition or jamming threshold [34-38], and it represents the onset of slow flow regime $[39,40]$, which seems to be rate-independent.

\section{The review of the proposed concept and related theory}

\subsection{Problem definition and hypothesis}

More than hundred years ago Reynolds [41,42] showed that dense sand expands at failure (which he named "dilatancy"), whereas loose sand contracts during shear to failure. Further research (mostly in soil mechanics) during last few decades shows that the direction of volume changes during shearing depends on the initial sand density at atmospheric pressure and the level of ambient applied pressure on the sand sample before shearing. Figure 1 presents the basic features:initially dense sand dilates during shearing in low pressure range and contracts at high pressures; initially loose sand contracts in almost whole pressure range except at very low pressures where it can also dilate. Also, it is found that, at large shearing deformations, the failure of sand sample takes place at constant volume, deviator and mean pressure (so called "critical state of sand", e.g., [30-33]). The relation between volume and pressure at critical state is virtually unique for the same sand and does not depend on initial conditions before shearing. This line, so called "critical state line" (CSL), also represents the borderline between contractive and dilative sand behavior. In Fig. 1 this line is shown as a projection on $p-v$ plane, while the common third coordinate is deviator

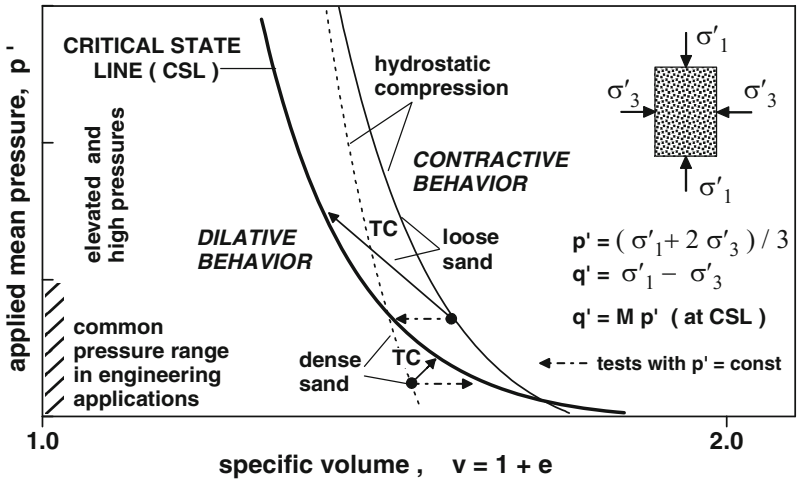

Fig. 1 Schematic summary of pressure-volume behavior in triaxial testing

stress $(q)$, which at CSS is not an independent variable. It is proportional to mean stress ( $p$ ) like $q=M p$, where $M$ is a constant reflecting the strength properties (internal friction) of sand body.

The observed pressure-volume behavior of sands during shearing shows inconsistencies with the pressure-volume behavior of common solids and fluids: the change of volume occurs without the change of pressure (for tests with constant mean pressure, where only deviator stress is applied), or, even more peculiar case: the expansion happens against the rise of compressive mean pressure (as in triaxial compression of initially dense sand).

Although the "stress-dilatancy" phenomena in sand have been the subject of several theories and models, it seems that clear and complete understanding of sand behavior (even in continuum approach) has not yet been found.

This was also the motivation for the proposal of simple constitutive model based on compressible fluid behavior [27] shown in Fig. 2. The proposed system consists of a cylinder with compressible fluid (e.g. gas) and a piston with constraint (e.g. "friction pads" with controlled force). The mechanical equilibrium of the system can be achieved for any volume of fluid $\left(v_{f}\right)$ in the cylinder when combination of forces from constraint $(F)$ and from pressure applied on external side of cylinder $\left(p_{a}\right)$ balances the pressure (force) inside the cylinder $\left(p_{f}\right)$. For the sake of simplicity, isothermal conditions are assumed, thus allowing the system to exchange the heat with environment during any process.

When applied to sands the role of constraint plays the friction ("internal friction of sand") which in the whole sand body is proportional to applied pressure. The shearing of sand can be understood as the process of "constraint release" in compressible fluid model.

The pressure-volume behavior of sands in Fig. 1 is presented from the position of applied external pressure and measured volume of sand samples. In the model from Fig. 2 this corresponds to $p_{a}$ and $v_{f}$, which are not uniquely related by fluid state equation because of external constraints on 


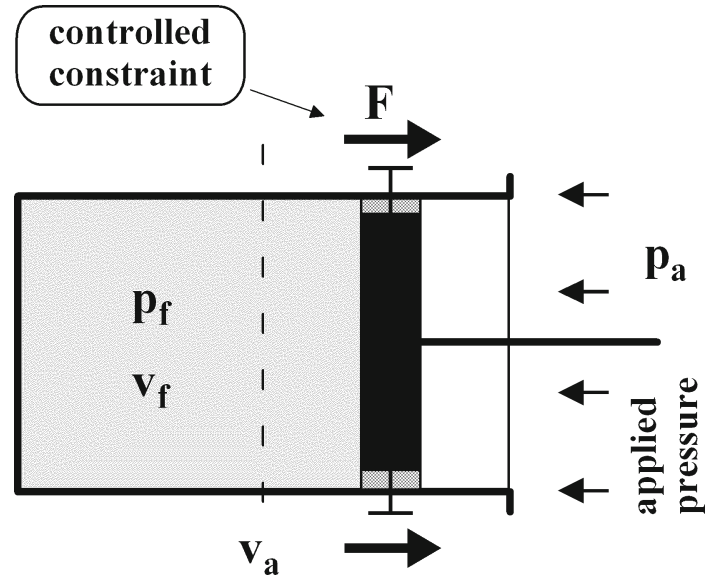

Fig. 2 The proposed model of compressible fluid for description of sand volume changes during shear [27]. For application on sands the weight of the sand body is formally added to the external actions. The resistance of sand body to shearing is understood as an analogue of external model constraint

"cylinder" (the corresponding pairs defined by state equation of fluids would be $p_{a}-v_{a}$ and $p_{f}-v_{f}$ ).

The hypothesis The above model presentation gave the idea for the hypothesis [27] that in the sand body also exists a thermodynamic (internal) pressure as in compressible fluids, defined by state equation of sand (state function) which uniquely relates pressure and volume, depending only on substance (mineral grains) properties. Consequently, from the suggested model and common knowledge on sand behaviour, this state equation would correspond to the "critical state line" of sand, where the effects of assumed "frictional constraint" are removed by shearing.

\subsection{The review of theory and previous findings}

The basic model analogy [27] between sand body and compressible, particularly van der Waals fluid, is shown in Fig. 3. The simple geometrical model uses continuum idealization of sand or fluid body, separated in compressible part (voids,

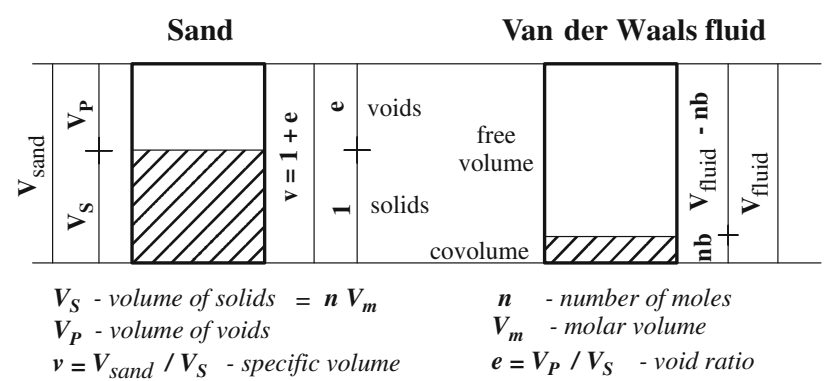

Fig. 3 The continuum idealization of volume relations in sand and van der Waals fluid. Note: The term "specific volume" differs in definition from the term with the same name in physical chemistry or thermodynamics. The above definition is common in soil mechanics free volume) and incompressible part (solids, covolume), and the adapted van der Waals equation is applied.

The van der Waals equation for $\mathrm{n}$ moles of real gas is written as

$\left(p+n^{2} a / V^{2}\right)(V-n b)=n R T$

where $p$-pressure, $V$-volume, $n$-number of moles, $R$ universal gas constant, $T$-temperature, $a$ and $b$-constants per mole of gas.

The adapted equation [27] is obtained when volume $V$ is normalized with the "incompressible" covolume $n b$, introducing dimensionless, relative or specific volume $v=V / n b$ (also shown in Fig. 3). Since $a, b$ and $R$ are constants, and $T$ is also assumed constant for sand analyses, the adapted equation can be written as

$\left(p+A / v^{2}\right)(v-1)=C$

where $A=a / b^{2}$ and $C=R T / b$, which is a suitable form for investigation of any system consisting of voids and matter like in Fig. 3, where measurable quantities, pressure and volume, are used (for isothermal conditions). This also applies to granular assemblies, or sands.

The new constants $A$ and $C$ have the units of pressure and are obtained empirically, for any set of corresponding measured data. The analysis of Eq. (2) for $p=0$ [27] shows that the obtained quadratic equation gives real values of volume (also comparable to empirical evidence) for the condition $A / C \geq 4$ with double solution $v=2$ for $A / C=4$.

Also, if Eq. (2) is extended in analogy to van der Waals fluids, it can be presented in reduced coordinates $(p, V, T$ normalized with corresponding values at critical point) to obtain the corresponding state. The diagram of "corresponding states" is presented in Fig. 4. The characteristic isotherm (reduced temperature, $T / T_{c}$ ) $\tau=27 / 32$ is found which corresponds to above mentioned conditions $A / C=4, p=0$ and $v=2$. The ordinary real values at critical point have no apparent meaning for mineral sands and the incompatibility is obvious for temperature effects (which are, also, the limits of analogy).

However, it was demonstrated [27] that the proposed analogy and application of Eq. (2) in reduced coordinates describes well the common soil mechanics tests in which the deviator stress is proportional to the mean pressure (also shown in Fig. 4).

\subsection{The interpretation of constants $C$ and $A$}

In order to extend the previous findings and analogy with the van der Waals fluid, in this paper the quantitative values of constants $C$ and $A$ are further examined for the case of "critical state of sand", where the hypothetic relations to substance properties can possibly be found. 


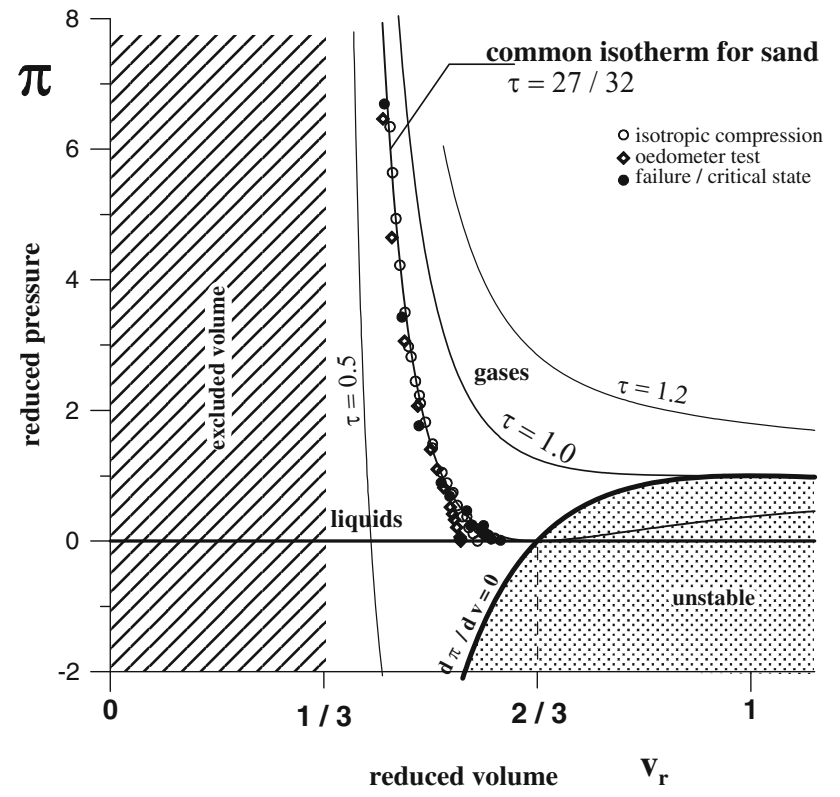

Fig. 4 The characteristic regions of van der Waals fluid in reduced coordinates - the diagram of "corresponding states", from [27]. For sands, the reduced pressure $\pi=27 p / A$, and the reduced volume $V_{r}=$ $v / 3$

The theoretical considerations for possible relations are outlined in following sections.

\subsubsection{The constant $C$}

Real gases In derivation of Eq. (2) the constant $C$ for mineral sands was assumed as a substitute for $R T / b$ in real gases. The product $R T$ is actually a product of pressure and volume of ideal gas $\left(p_{0} V_{0}\right)$ in normal conditions, corrected for thermal expansion (since $R=p_{0} V_{0} / T_{0}$ and $T / T_{0}=\left(T_{0}+\right.$ $\Delta T) / T_{0}=1+\alpha_{v} t, \alpha_{v}$-thermal expansion coefficient of ideal gas, $T_{0}=273.15 \mathrm{~K}=0{ }^{\circ} \mathrm{C}$, $t$-temperature in ${ }^{\circ} \mathrm{C}$ ). The volume excluded by a mole of particles, $b$, has an upper bound of four times the proper volume of solid particles, $V_{m}$, as originally derived by van der Waals, assuming particles as hard spheres.

Mineral sands The constant $C$ for sands was partitioned similarly as for gases using the following considerations:

1. Ordinary temperature plays no particular role in granular matter, and in a wide range of ordinary temperatures no significant thermal effects in sands can be observed. Also, thermal expansion of sand is negligible in a common range of temperatures, since the thermal expansion coefficient for mineral grains is about one percent, or less, of the ideal gas coefficient. The experimental work described above was done at usual laboratory temperature, and the assumption of constant temperature for sand analyses also means that isothermal conditions have been analyzed.
In order to allow for thermal (or other non-assumed) effects, the empirical factor $\beta_{S}$ is introduced as a substitute for $T / T_{0}$ in former gas constant derivation.

2. The volume of solid particles in sand samples is proportional to their molar volume $V_{m}$ (from Fig. $1: V_{S}=$ $n V_{m}, n$-number of moles). The molar volume $V_{m}$ of mineral materials is obtained from macroscopic specific density of mineral solids, $\rho_{S}$, and molar mass, $M_{m}$ (for basic reported dominant constituent of tested materials), like $V_{m}=M_{m} / \rho_{s}$. The density of mineral grains at laboratory temperature is approximately the same as for $\mathrm{t}$ $=0^{\circ} \mathrm{C}$ (very low thermal expansion of minerals), thus $V_{m} \approx V_{m 0}$. The substitute for excluded volume $b$ in real gases was taken as $4 V_{m}$, to comply with vdW fluid..

The final structure of constant $C$ for sands using the considerations above is:

$C=\beta_{S} p_{0} V_{0} / b=\beta_{S} p_{0} V_{0} / 4 V_{m}$

or, more specific

$C=\beta_{S} p_{0} V_{0} \rho_{S} / 4 M_{m}$

However, allowing for other possible effects and assumptions, taking into account that the real excluded volume in sands is only the volume of solids $V_{S}$ or $n V_{m}$ for $n$ moles of sand (since all the voids between the grains are taken into account), and also allowing for experimental errors, more general parameter $\gamma_{S}$ can be introduced.

The parameter $\gamma_{S}=\gamma_{S}(n, m, x, z \ldots)$ is understood as a free parameter allowing for further empirical studies of its possible dependence on any other factor affecting the sand properties at critical state.

The more general expression for the constant $C$ becomes:

$C=\gamma_{S} p_{0} V_{0} / V_{m}$

\subsubsection{The constant A}

Real gases In derivation of Eq. (2) the constant $A$ was the substitute for real gas constants $a$ and $b$ as $A=a / b^{2}$. These constants for gases are usually obtained from the critical point using the critical pressure, $p_{c}$, critical temperature, $T_{c}$, and critical volume $V_{c}$. Using known expression such as $p_{c}=$ $a / 27 b^{2}$, it is obvious that the constant $A$ in real gases is a multiple of critical pressure i.e. $A=27 p_{c}$.

The other known expression involves critical temperature: $T_{c}=8 a / 27 b R$.

Rewriting this expression and dividing both sides with $b$, one obtains:

$a / b^{2}=27 R T_{c} / 8 b$

or

$A=(27 / 8)\left(T_{c} / T_{0}\right)\left(p_{0} V_{0} / b\right)=K_{G}\left(p_{0} V_{0} / b\right)$ 
where the relation of $A$ to $\left(p_{0} V_{0} / b\right)$ is emphasized and the other part of Eq. (7) (signed as $K_{G}$ ) also presents the individual constant, including the ratio of critical and normal temperature.

Also, if the constant $C=R T / b$ is written for characteristic "sand" temperature $T=27 T_{c} / 32$, a similar expression for $C$ is obtained:

$$
C=R T / b=27 R T_{c} / 32 b=(27 / 32)\left(T_{c} / T_{0}\right)\left(p_{0} V_{0} / b\right)
$$

For this particular isotherm the constant $C=A / 4$, showing the same relation to other material constants.

Mineral sands In the interpretation of sand tests using Eq. (2), the constant $A$ is obtained from the same set of data as the constant $C$ and represents the intercept of the line fitting this data. As discussed earlier, the ratio $A / C$ should be $A / C \geq 4$ to satisfy the stable conditions using van der Waals equation, and, also, the condition $A / C=4$ complies with the characteristic isotherm $\tau=27 / 32$ in reduced coordinates.

The presented interpretations of test results demonstrate that empirically obtained $A / C$ ratio is close to 4 . Since the same data sets (coming from the same origin) are used to obtain both constants $C$ and $A$ for sands, the above derivation of constant $C$ can be applied to constant $A$ as:

$A \approx 4 C \approx 4 \beta_{S} p_{0} V_{0} / b \approx \beta_{S} p_{0} V_{0} / V_{m}$

Or, to comply with Eq. (5):

$A=(A / C) * C=\gamma_{S}(A / C) p_{0} V_{0} / V_{m}$

generally showing and emphasizing the relation of $A$ to $\left(p_{0} V_{0} / V_{m}\right)$.

\section{Experimental results and interpretation}

\subsection{Testing procedure}

The basic test for this investigation of pressure-volume relations is the triaxial test, common in experimental soil mechanics $[30-33,43,44]$. The most common procedure is "triaxial compression", when the isotropically compressed sample (to a certain value of cell pressure) is sheared until failure by raising vertical stress and keeping lateral stress constant. At large shearing deformations the failure of sand sample takes place at a constant volume, deviator and mean pressure (CSS). This failure state of the sand sample is also the end of the common individual triaxial test. The series of these tests, starting from different lateral (or cell) pressures (on the same material), yield a virtually unique "failure line" or "critical state line" (CSL), connecting mean pressures, deviator stresses and volumes of sample at the end of the individual tests. The results (mean pressures and volumes) of a series of tests and the course of some tests are illustrated

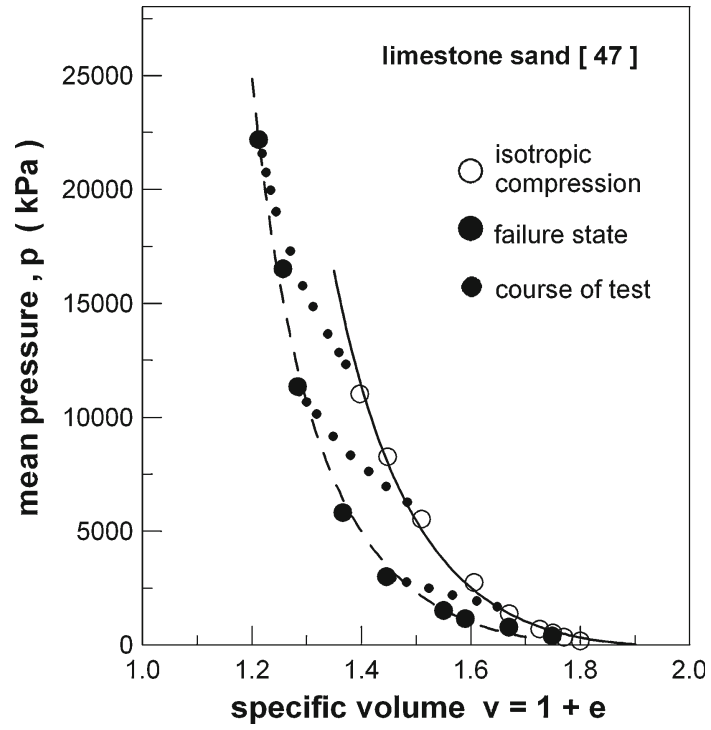

Fig. 5 Relevant test results from the common triaxial compression test

in Fig. 5. The exact conditions of critical state cannot be easily reached in all common tests, since the large deformations (slow flow of sand) cause mechanical instabilities. So, most of presented results demonstrate the state close to critical.

The volume changes of saturated samples are usually established by measuring the volume of pore fluid (e.g. deaired water) draining from the voids in the sample during compression. Also, other techniques can be used for tests on dry samples $[44,45]$.

\subsection{Interpretation procedure of test results}

The basic test results at reported failure (mean pressure and volume) presented in this work are interpreted in order to get numerical values of constants $C$ and $A$ from Eq. (2). The auxiliary coordinates $f_{p}=p v^{2}$ and $f_{v}=v^{2} /(v-1)$, which are both the functions of measured quantities, came from the adapted van der Waals equation, Eq. (2). The reinterpretation of this equation [27] leads to the linear relation between $f_{p}$ and $f_{v}$ as:

$f_{p}=C f_{v}-A$

in which constant $C$ is the slope of the line, and constant $A$ is the intercept. The ratio $A / C$ can be directly read on the $f_{v}$ - axis for $p$ (or $f_{p}$ ) $=0$. The average line through the test points is found by simple linear regression.

The example of interpretation of tests from Fig. 5 using above procedure is presented in Fig. 6 .

\subsection{Materials and test conditions}

In order to cover a wider range of materials and test conditions, the published results of the triaxial test series were 


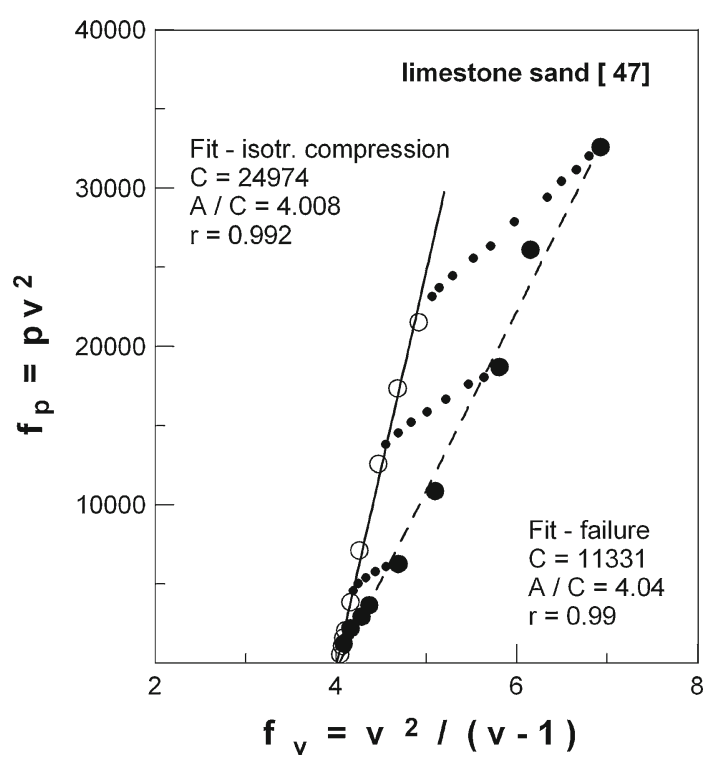

Fig. 6 Interpretation of test results from Fig. 5 using Eq. (11)

used [44,46-52]. The test results are graphically presented in Electronic Supplement material. ${ }^{1}$ The testing details are presented in the corresponding references and some basic data are mentioned in Supplement to illustrate the interpretation of the test series.

The test series included sands composed of different minerals (quartz, limestone, kaolin clay and quartz-clay mixture) from various locations in the world. The presented collection of tests also covers a wide range of applied confining pressures and initial void ratios.

\subsection{The interpretation of tests and comments}

\subsubsection{The results of test interpretation}

The results of interpretation using simple linear regression analysis for Eq. (11) are summarized in Table 1. for individual tests The tests have also been interpreted for the condition $A / C=4$, which shows compliance with common, characteristic isotherm for sands ( $\tau=27 / 32$ in reduced coordinates) described earlier.

The later interpretation was also the only interpretation for the situations where only a few tests in narrow pressure range have been analyzed. It was not used only for Portaway sand [52], since the large initial volume shift made this interpretation unrealistic. For most of the other test series these two interpretations gave similar results for constant $C$, since the $A / C$ ratio obtained from linear regression was close to

\footnotetext{
${ }^{1}$ See Electronic Supplement Material for: A. The results of triaxial tests for the "critical state of sands"; B. Van der Waals constants and critical temperatures for gases and volatile fluids.
}

4. The illustration example for this double interpretation is in Fig. 7.

Since the analyzed test series were performed in the pressure range of several magnitudes it is impractical to present all the results in one diagram using auxiliary coordinates $f_{p}$ and $f_{v}$.

In order to compile all results in a single plot, the Fig. 8a$\mathrm{b}$ are presented using normalized coordinate $\left(f_{p}+A\right) / C$ versus $f_{v}$ and best fits for $C$ and $A$ from individual test series. Since many results in lower range overlap, Fig. $8 \mathrm{~b}$ presents the narrower range of coordinates in order to distinguish individual test data.

The data for all tests lie close to 1:1 line what demonstrate the compliance with function defined by Eq. (2). The scatter is pronounced for high pressure tests on Chattahoochee River Sand [46] which is commented below.

The individual constants reflect the mineral composition which is also illustrated in Fig. 7, where marked difference of line slopes (constant $C$ ) is evident for quartz and kaolin clay.

\subsubsection{Comments on tests}

The fits comply with adapted van der Waals equation, Eq. (2) within the experimental accuracy. The scatter of results is visible in figures (see footnote 1), and the indicator of "goodness of fits" is also given in Table 1. by coefficient of determination, $r^{2}$, from simple regression analyses, which is usually described as the "measure of the proportion of variability in a data set that is accounted for by a statistical model". Generally, the fits are better in a larger pressure range.

The test conditions significantly influence the scatter and the consistency of results. The comprehensive review of experimental details, techniques and difficulties in measurement of critical state of sand in triaxial tests is presented by Jefferies and Been [44].

The proposed Eq. (2) is of the 3rd order and very sensitive to input volume values, so the accuracy of volume measurement is essential. This reason can be attributed to the scatter for large pressures on Chattahoochee River Sand [46], since the volume for dry sand had been measured outside the sample, approximating the sample as a barrel. Tests on the same sand, where volume was measured by expelled fluid (series C, D), are more consistent.

The scatter of all results for Erksak sand [44] may be diminished when only the tests with identical testing conditions are used. Although some inconsistencies can be found between individual test results, there was no particular strong criterion to discriminate certain tests. The exceptions are the tests in very low pressure range, which show sharp deviation. This deviation is consistent with suggestions in literature [44], that initially dense sand at low pressures is not representative for CSL measurements, due to dilatancy 
Table 1 Summary of triaxial test series interpretation for critical state of sand

\begin{tabular}{|c|c|c|c|c|c|c|c|c|c|}
\hline Sand no. & Sand name & Mineral & Ref. & Remark & Number of points & $C\left(\mathrm{kN} / \mathrm{m}^{2}\right)$ & $A\left(\mathrm{kN} / \mathrm{m}^{2}\right)$ & $A / C$ & $r^{2}$ \\
\hline \multirow[t]{2}{*}{1} & \multirow[t]{2}{*}{-} & \multirow[t]{2}{*}{ Limestone } & \multirow[t]{2}{*}{47} & All tests & 9 & $11,331.3$ & \multirow[t]{2}{*}{$45,775.5$} & \multirow[t]{2}{*}{4.04} & 0.990 \\
\hline & & & & $\mathrm{A} / \mathrm{C}=4$ & 9 & $11,097.1$ & & & 0.990 \\
\hline \multirow[t]{2}{*}{2} & \multirow[t]{2}{*}{-} & \multirow[t]{2}{*}{ Quartz } & \multirow[t]{2}{*}{47} & High pressure results & 4 & $46,313.9$ & \multirow[t]{2}{*}{$176,939.0$} & \multirow[t]{2}{*}{3.82} & 0.953 \\
\hline & & & & $\mathrm{A} / \mathrm{C}=4$ & 4 & $55,844.0$ & & & 0.904 \\
\hline \multirow[t]{4}{*}{$3 a$} & \multirow[t]{6}{*}{ Chattahoochee } & \multirow[t]{6}{*}{ Quartz } & \multirow[t]{6}{*}{46} & All tests & 40 & $34,222.7$ & \multirow[t]{2}{*}{$135,617.0$} & \multirow[t]{2}{*}{3.963} & 0.787 \\
\hline & & & & $\mathrm{A} / \mathrm{C}=4$ & 40 & $34,610.4$ & & & 0.787 \\
\hline & & & & Only dry samples & 22 & $32,086.3$ & \multirow[t]{2}{*}{$115,676.0$} & \multirow[t]{2}{*}{3.605} & 0.624 \\
\hline & & & & $\mathrm{A} / \mathrm{C}=4$ & 22 & $35,660.0$ & & & 0.614 \\
\hline \multirow[t]{2}{*}{$3 b$} & & & & Only $p=$ const & 18 & $20,387.5$ & \multirow[t]{2}{*}{$80,001.8$} & \multirow[t]{2}{*}{3.924} & 0.897 \\
\hline & & & & $\mathrm{A} / \mathrm{C}=4$ & 18 & $21,228.0$ & & & 0.892 \\
\hline \multirow[t]{2}{*}{4} & \multirow[t]{4}{*}{ Cambria } & \multirow[t]{5}{*}{ Mixed } & \multirow[t]{4}{*}{48} & Range $\mathrm{p}_{f}: 0-22 \mathrm{MPa}$ & 11 & $11,422.7$ & \multirow[t]{2}{*}{$42,211.9$} & \multirow[t]{2}{*}{3.695} & 0.989 \\
\hline & & & & $\mathrm{A} / \mathrm{C}=4$ & 11 & $13,556.2$ & & & 0.945 \\
\hline \multirow[t]{2}{*}{$4 a$} & & & & Range $\mathrm{p}_{f}: 0-65 \mathrm{MPa}$ & 19 & $16,489.4$ & $67,673.6$ & 4.104 & 0.942 \\
\hline & & & & $\mathrm{A} / \mathrm{C}=4$ & 19 & $15,783.8$ & & & 0.940 \\
\hline $4 b$ & & & & $\mathrm{p}_{f}>65 \mathrm{MPa}, \mathrm{A} / \mathrm{C}=4$ & 3 & $27,653.7$ & & & 0.646 \\
\hline 5 & Sydney & Quartz & 50 & Without test No\#5 & 7 & $57,199.5$ & $230,838.0$ & 4.036 & 0.977 \\
\hline & & & & $\mathrm{A} / \mathrm{C}=4$ & 7 & $36,961.7$ & & & 0.833 \\
\hline 6 & - & Kaolin & 51 & All tests & 14 & $2,393.7$ & $9,741.2$ & 4.07 & 0.938 \\
\hline & & & & $\mathrm{A} / \mathrm{C}=4$ & 14 & $1,953.43$ & & & 0.902 \\
\hline 7 & Erksak & Quartz & 44 & All tests, $\mathrm{p}_{f}>200 \mathrm{kPa}$ & 24 & $27,250.4$ & $109,420.0$ & 4.015 & 0.753 \\
\hline & & & & $\mathrm{A} / \mathrm{C}=4$ & 24 & $25,157.9$ & & & 0.748 \\
\hline & & & & Only pluviated & 6 & $23,131.6$ & $92,001.6$ & 3.977 & 0.624 \\
\hline & & & & $\mathrm{A} / \mathrm{C}=4$ & 6 & $25,520.8$ & & & 0.617 \\
\hline & & & & Moist tamping, $\mathrm{A} / \mathrm{C}=4$ & 3 & $21,779.1$ & & & 0.758 \\
\hline & & & & High pressure apparatus & 7 & $34,605.2$ & $139,184.0$ & 4.022 & 0.846 \\
\hline & & & & $\mathrm{A} / \mathrm{C}=4$ & 7 & $30,493.7$ & & & 0.833 \\
\hline 8 & Portaway & Quartz & 52 & All tests, $\mathrm{p}_{f}>70 \mathrm{kPa}$ & 20 & $23,126.6$ & $95,756.5$ & 4.14 & 0.807 \\
\hline
\end{tabular}

effects. The lower pressure test results were excluded from analyses and the limiting pressure was chosen arbitrarily, with the intention of using as many test results as possible.

The authors believe that that the triaxial compression is not the best suited test for achieving the critical state of sand. The necessary conditions of free, slow flow of sand would require different test equipment, where the sand sample is not confined and where the shearing can be maintained within a large range of pressures with accurate volume measurements (perhaps like in some modified ring shear tests, or equipment with moving boundaries).

\subsubsection{The constant $C$}

In the interpretation of the constant $C$ the Eq. (4) and empirical correction factor $\beta_{S}$ were used, for convenience, and to comply more with van der Waals fluid.

The values of constant $C$ for typical minerals used in experiments obtained by Eq. (4) with $\beta_{S}=1$ are presented in
Table 2. Also presented is the possible expected range of values for the mineral mixture Cambria sand [48,49], which is reported as predominantly quartz with clay (shale) grains. For this purpose, the estimate of weighted average molar mass was made for composition of quartz and kaolin, assumed as a representative of clay minerals.

The results for constant $C$ in triaxial tests are compared with Eq. (4) for $\beta_{S}=1$ in Fig. 9a. The range of constants from Table 1 for particular sand is positioned at the value of the calculated molar covolume, assuming that the real sands were composed of $100 \%$ the dominant mineral reported. The Cambria sand is presented in the estimated range of molar volumes and medium pressure range.

In Fig. 9b the ratio of constants in tests and Eq. (4) for $\beta_{S}=1$ is shown, giving the value of empirical coefficient $\beta_{S}$ for a particular test.

The basic observation from the figures is that Eq. (4) predicts well the order of magnitude of constant $C$ for mineral sands even for $\beta_{S}=1$. This also means that the dominant 


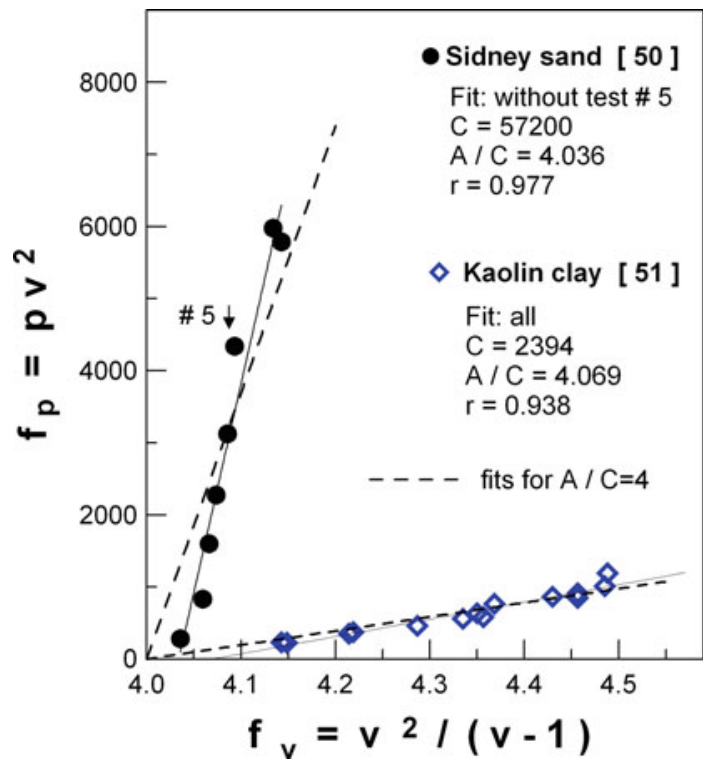

Fig. 7 Interpretation of test results using fits for all test data and for the condition $A / C=4$

quantitative parameters in constant $C$ are molar properties of minerals and ideal gas.

Most of the quartz results give $\beta_{S}$ close to 1 , with few extremes above. The results for limestone and Cambria (as a crude estimate) are not far from $\beta_{S}=1$, and the single result for kaolin is the lowest giving $\beta_{S}=0.35-0.4$. The deviation of constant $C$ for Cambria sand in very high pressures range quantitatively complies with expected value for quartz (see, Table 1).

The results also demonstrate the expected trend of lowering of constant $C$ for greater mineral molar volumes. For minerals other than quartz only single test series have been collected and no further conclusions can be drawn from this interpretation.

Also, it is to be noted that the test results cover the measurement technologies during half a century, and that the triaxial test is a very cumbersome way for obtaining CSS conditions. In the way presented, the variations of coefficient $\beta_{S}$ also reflect possible experimental errors.
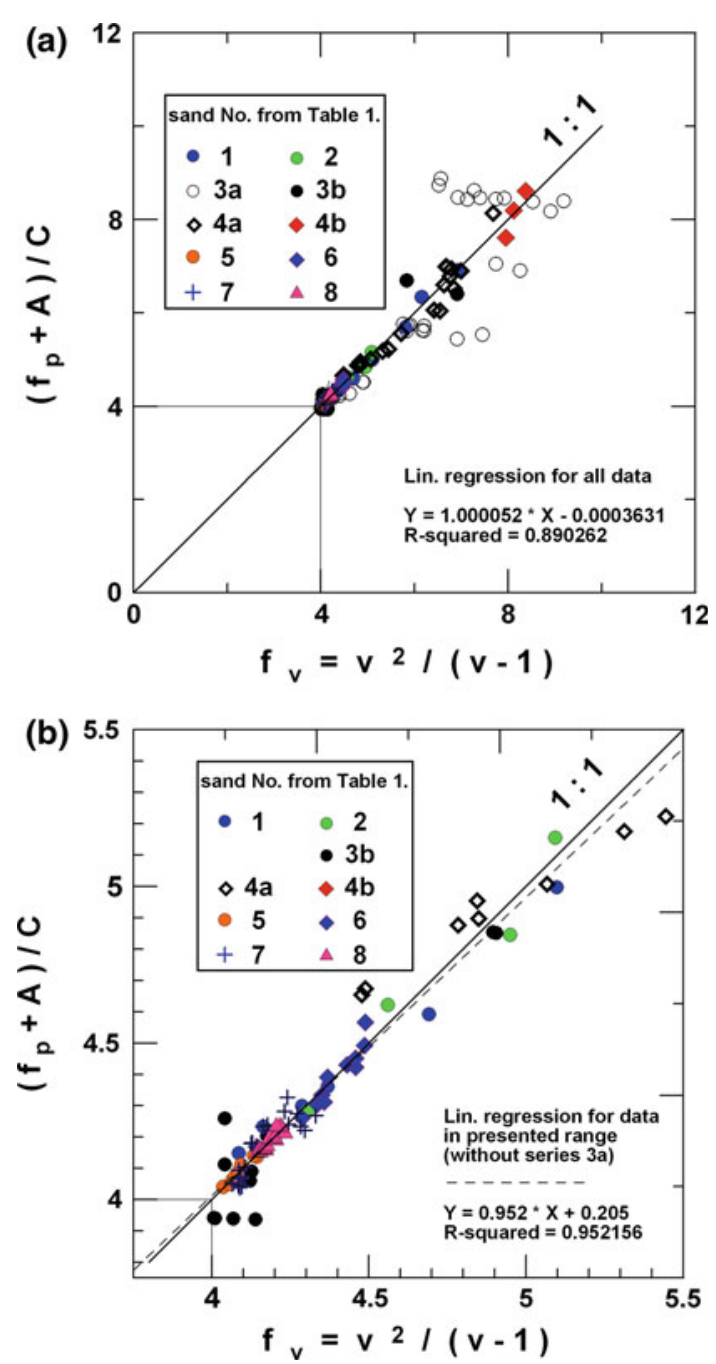

Fig. 8 Presentation of test data in normalized coordinates: a all data; b data for lower pressures

\section{The relation between real fluids and sands}

\subsection{The constant $A$}

As demonstrated in Sect. 2.3.2 the constant $A$ is an empirical material constant (composed of common van der Waals con-

Table 2 Summary of constant $C$ for tested minerals estimated using Eq. (4) and $\beta_{S}=1$

\begin{tabular}{llllll}
\hline Mineral & Used formula & Sp.density $\left(\mathrm{t} / \mathrm{m}^{3}\right)$ & Molar mass $(\mathrm{kg} / \mathrm{kmol})$ & $4 V_{m}\left(\mathrm{~m}^{3} / \mathrm{kmol}\right)$ & $\mathrm{Constant} C$ as $\left(p_{0} V_{0} / 4 V_{m}\right)^{\mathrm{a}}$ \\
\hline Quartz & $\mathrm{SiO}_{2}$ & 2.65 & 60 & 0.0906 & 25,077 \\
Limestone & $\mathrm{CaCO}_{3}$ & 2.71 & 100 & 0.1476 & 15,387 \\
Kaolin & $\mathrm{Al}_{2} \mathrm{Si}_{2} \mathrm{O}_{5}(\mathrm{OH})_{4}$ & 2.60 & 258 & 0.397 & 5,722 \\
Cambria (possible range) & $55 \mathrm{Q}+45 \mathrm{KA}$ & 2.69 & $149.1^{\mathrm{c}}$ & 0.2217 & 10,244 \\
& $65 \mathrm{Q}+35 \mathrm{KA}$ & 2.69 & 129.2 & 0.1923 & 11,812 \\
& $75 \mathrm{Q}+25 \mathrm{KA}$ & 2.69 & 109.5 & 0.163 & 13,948 \\
\hline
\end{tabular}

${ }^{\mathrm{a}}$ With $p_{0}=101.325 \mathrm{kPa}, V_{0}=22.414 \mathrm{~m}^{3} /$ kmole

${ }^{\mathrm{b}} 55 \mathrm{Q}$ - mass percentage of quartz, $45 \mathrm{KA}$-mass percentage of kaolin

${ }^{\mathrm{c}}$ Weighted average molar mass 

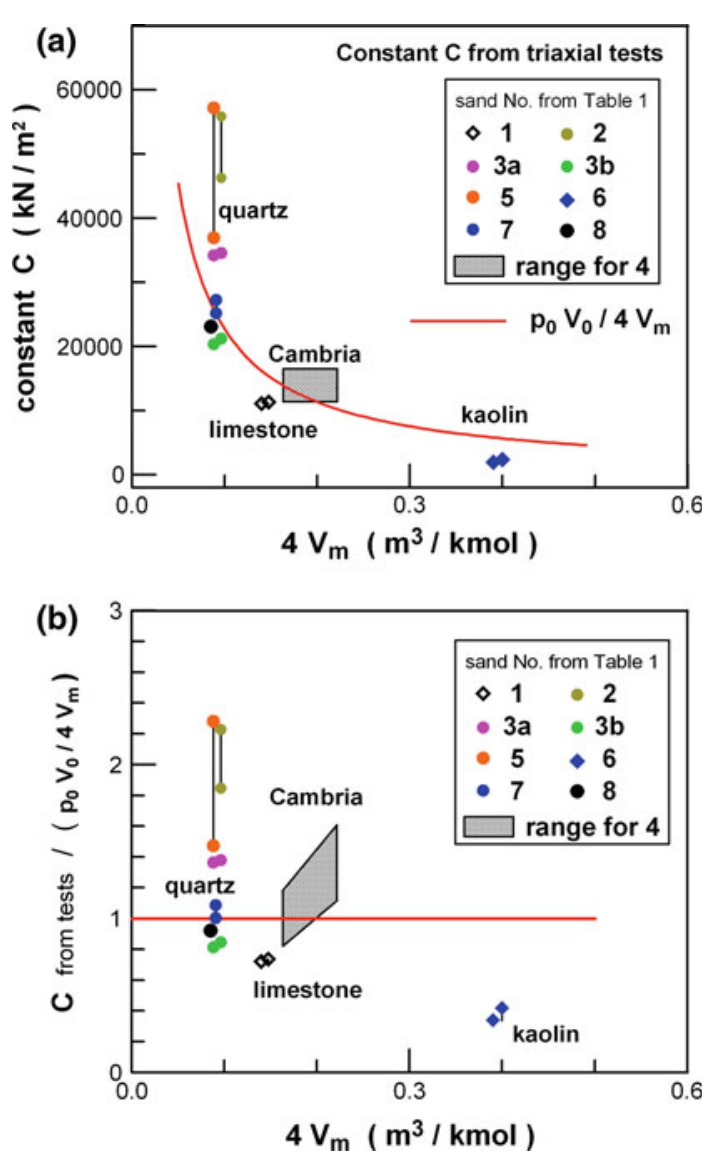

Fig. 9 The comparison of constant $C$ from triaxial tests with the estimates from Eq. (4), $\mathbf{a}$ for $\beta_{S}=1, \mathbf{b}$ the estimate of $\beta_{S}$

stants $a$ and $b$ ) which, according to outlined theory, enables direct quantitative comparison of corresponding substance features of real fluids and mineral sands.

The published van der Waals constants for about 60 gases and volatile liquids have been collected and roughly classified as elements, organic and anorganic compounds in supplement material (see footnote 1). The general trend is an approximately linear rise of the constant $a$ with excluded volume $b$, with a few exceptions and some scatter.

In Fig. 10 the constant $A$ for those fluids is presented using the above constants $a$ and $b$. The scatter of results is significant mostly for elements and anorganic compounds. However, most of the results fall in the range of $A=100,000$ $200,000 \mathrm{kPa}$.

Also presented are the results from sand triaxial tests for the expected macroscopic molar volume of dominant mineral. For simplicity, only the results for $A / C=4$ are shown. The results for sands are somewhat lower than for fluids, but still comparable and within range of empirical values.

The first part of Eq. (7) signed as $K_{G}$ was calculated using published values of critical temperature for particular fluid (presented in Supplement material - see footnote 1). These

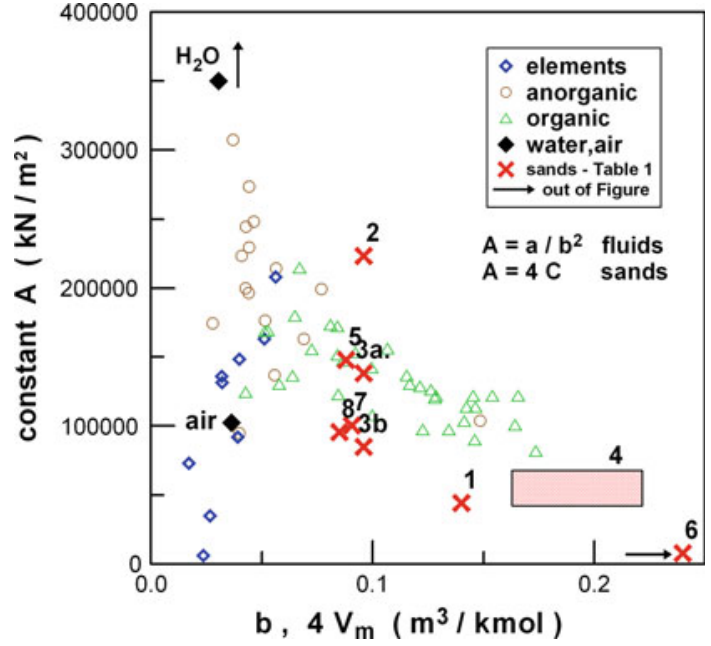

Fig. 10 The constant $A=a / b^{2}$ for gases and $A=4 C$ for sands

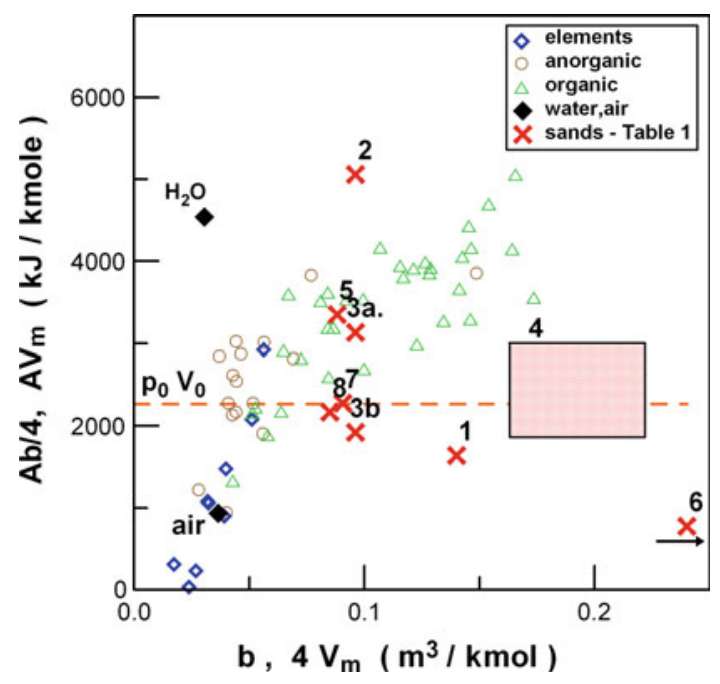

Fig. 11 The attraction energy interpreted from vdW constants

values show that this "correction factor" for fluids to relate constant $A$ to $\left(p_{0} V_{0} / b\right)$ ranges from 0.06 to 9 , and the scatter of results reflects the scatter of individual critical temperatures . As demonstrated in Fig. 9, the corresponding "correction factor", $\beta_{S}$, for sands is of the order of 1 .

\subsection{Attraction energy}

The van der Waals constant $a$ is a measure of attraction between the particles in real gases, and a new constant $A$ (expressed as pressure in $\mathrm{kPa}$ ) could also be understood as the attraction energy density (energy per volume, expressed in $\mathrm{kJm}^{-3}$ ). When the proper volume of molecules in real gas is used (which is about $b / 4$ ), the energy per mole of matter becomes $A(b / 4)=a / 4 b$. These values are shown in Fig. 11 .

The approximate expected common range of values for weak noncovalent interactions attributed to van der Waals 
forces $\left(<5 \mathrm{~kJ} \mathrm{~mol}^{-1}\right.$ or MJ kmole $\left.{ }^{-1}\right)$ e.g. [53], is found for the analyzed fluids.

The application on sands using molar volume $V_{m}$ and Eq. (9) leads to expected "sand energy" of

$A * V_{m}=\left(\beta_{S} p_{0} V_{0} / V_{m}\right) * V_{m}=\beta_{S} p_{0} V_{0}$

which has the value of $2.271 \beta_{S} \mathrm{~kJ} \mathrm{~mol}^{-1}$ (or $\mathrm{MJ} \mathrm{kmol}^{-1}$ ). For $\beta_{S}=1$ this value is "in the middle" of the results for fluids.

Apparent attraction and repulsion in sands The apparent attraction coming from constant $A$ does not seem to be verified as an observable attraction between grains in real sand bodies like hourglass, quicksand or desert dunes. Also, it does not seem very likely that the common known van der Waals forces between gas molecules could be applied to mineral sand grains. These forces decay with distance on the molecular scale and they would totally vanish far within the single sand grain.

Although the ordinary van der Waals forces are assumed to act on molecular scale, there are attempts to extend the range of these forces to powder or grain scale and to correlate macroscopic features to those forces [54]. In clay soils, where most of the particles are of colloid size the underlying mechanism for changes of the macroscopic, engineering properties is usually attributed to combined effect of repulsion/attraction between clay particles (DLVO forces) or surface charge effects [55].

Mineral sand grains, which are much larger than colloids, appear to be non-attractive or cohesionless. It is, however, interesting that on the macroscopic level the above analogy between fluids and sands gave the quantitative order of magnitude for attraction energy, at least as a bulk property (per mole of matter).

In presentation of van der Waals equation for gases, the parts of equation related to repulsion $(R P)$ and attraction $(A T)$ are often recognized. Also, the pressure (or energy density) can be presented as:

$p=R P-A T$

When the form of adapted Eq. (2) is used, the attraction and repulsion functions become $A T=A / v^{2}$ and $R P=C /(v-$ $1)$. These attraction and repulsion functions are presented in Fig. 12 separately as positive functions of specific volume for the case $A / C=4$. The functions are normalized with constant $C$.

For the extreme case without voids i.e., $v=1$, the attraction function has a finite value while the repulsion function, as well as the pressure, tends to infinity.

The specific case is for $v=2$ (or the case where half of the total volume is occupied by solids and half is in voids), where the attraction and repulsion are in balance and the pressure is zero. For larger volumes the attraction decays faster than repulsion (with the square of volume) and the pressure slowly

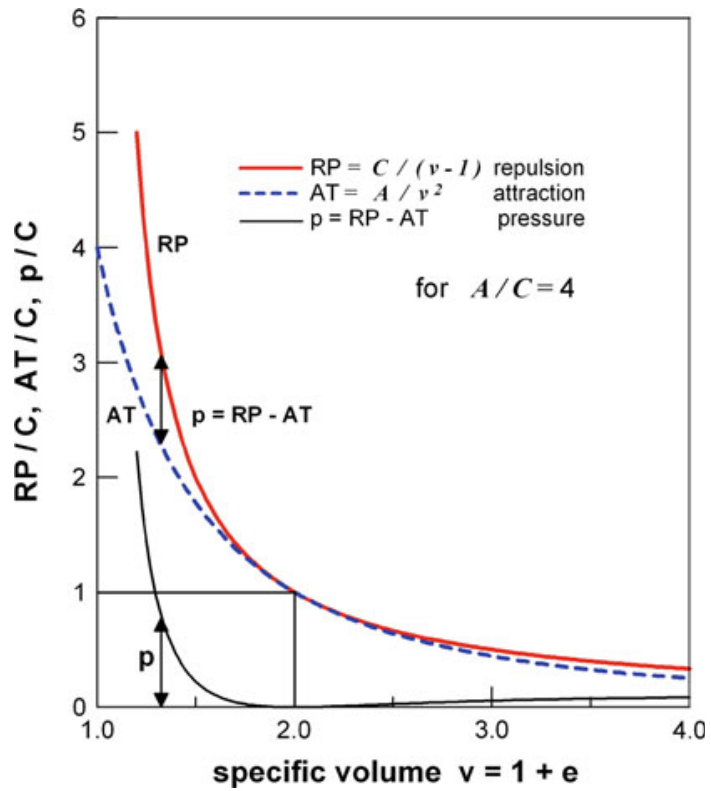

Fig. 12 Attraction and repulsion in vdW body, functions from Eq. (11)

rises with volume. Volumes greater than 2 theoretically [27] do not represent the stable state of sands.

It seems generally acceptable that the pressure (or some internal energy density) in a body is the net, overall effect of repulsion and attraction forces. Since, for the presented case, the attraction is always lower than repulsion, the apparent net internal behavior of sand would be inherently repulsive and the balance in nature for particular sand volume could be obtained by gravity or otherwise applied pressure. This is not in contradiction with macroscopic appearance of dry sands, and satisfies the constitutive model from Fig. 2.

However, at this level of information it is not quite clear what would be the nature of these apparent attractive/ repulsive forces in a bulk of sand grains. It seems that above discussion is more an indication than an explanation.

\subsection{Discussion and observations}

General observations on the presented analogy Granular materials are often understood as assemblies of discrete solids whose behavior is, in general, governed by interparticle friction, contact forces, collisions and geometry (fabric). Since the thermal effects are irrelevant, it is understood that the ordinary thermodynamic arguments become useless [2].

The above finding, that the analogy to real gas behavior can be enhanced using the ideal gas concept and molar properties of material, is quite unexpected. Still, the order of magnitude for corresponding constants is achieved, and dominant quantitative term in constants is $p_{0} V_{0} / V_{m}$.

It is also to be noted that the empirical van der Waals constants are also basically independent of temperature and 
represent the intrinsic properties of matter, which is not in contradiction with proposed analogy.

The presented analogy between sand body and common compressible fluids is based on a few similar features as described in chapter 2 . The agreements and non-contradictions that are found might be encouraging and some of them are discussed here, but there are still many limits and differences to prevent the physical explanation of these agreements in the way used for common fluids.

Despite the analogies, the gases and sands in reality appear as significantly different systems. The experimental way of obtaining corresponding empirical constants is completely incomparable: the constants for gases are usually obtained from critical variables involving temperature conditions, while for particular, relevant state of sand, the mechanical isothermal tests have been used.

On the other side, it may be noticed that in gaseous phase the molecules are separated and not in the domain of strong molecular interactions. When the set of molecules is understood purely geometrically as a bulk of packed particles, the molecules in that phase still satisfy main assumptions of proposed bulk, continuum idealization (incompressibility and finite volume of matter).

The effects of gravity In the model from Fig. 2, the weight of grains alone is assumed as an external action. In common sand tests the pressures only due to the weight of sand sample are negligible when compared to the externally applied pressures. Also, in common soil mechanics interpretation, these tests are understood as a simulation of isolated control volume at certain depth in real sand masses. This sample/control volume is loaded by above layers or any other external load and laterally confined by the rest of the sand mass.

However, the gravity is the volume force and possible source of grain interactions. The above assumption, that the effects of grain weight are negligible, and that they affect the sand sample in the same way as the external actions, can be tested in variable gravity conditions, possibly in geotechnical centrifuge.

Pressure-volume relations in sands The pressures at the critical state of sand are understood as the best representative for the applied analogy, since this state appears to be unique in soil testing and in appearance nearest to the fluids (state of failure of sand body and beginning of slow flow at constant volume).

The hydrostatic (isotropic) compression tests on sands seem to be more similar to the testing conditions of common compressible fluids. As shown earlier [27] and in Figs. 5 and 6 , the pressure-volume behavior from these tests can generally also be described by proposed Eq. 2, but with different values of empirical constants than for critical state.

Since many functional $p-V$ relations can be found for isotropic loading conditions, request for uniqueness is questionable. There are still many disputes about the "loosest state of sand" in low ambient pressure whose $p-v$ behavior in isotropic conditions could define the unique "state boundary". The proposed van der Waals model suggests that the loosest possible stable state for zero pressures occurs for the conditions $v=2$ (or porosity $50 \%$ ). Eventually looser state at near zero pressures could be attributed to additional, structural forces like capillarity, cementation or other surface forces.

Voidage In derivation of adapted van der Waals Eq. (2) the volume of solids in sand and its analogous part in real gasescovolume $b$ are taken as normalizing parameters representing constants and incompressible part of the idealized model from Fig. 1. This normalization emphasizes the separation of total volume to voids and solids.

The common interpretation in soil testing is that the volume changes are attributed only to the changes of voidage [7,30-33,43]. It appears the same in real gases since the volume of molecules included in constant $b$ basically does not change. Even the thermal expansion affects only the free volume of gases to obtain the total volume changes.

The effect of voidage is not so obvious in macroscopic gas bodies, where the total volume is measured and taken as a variable. In soils, the changes of voidage are measured more directly by the volume of pore fluid (water or air) which is expelled or soaked to sand sample during loading. Also, the external measurements on sand sample can be performed to geometrically obtain the sample volume changes.

Grain crushing The large compression and shearing of sands is associated with grain crushing [56-58], which significantly alters the initial structure of sand sample during tests. It appears that this effect does not influence the pressure-volume behavior presented here for mineral sands.

The mass of the sand grains, $M_{S}$, during particular test is constant and the specific density of minerals, $\rho_{S}$, is usually obtained for already crushed (tiny) particles. This means that the volume of solids, $V_{S}$, is also constant during test (since $V_{S}=M_{S} / \rho_{S}$ ), and (referring to Fig. 3) the volume changes of sand sample are attributed only to the changes in volume of voids (which, in bulk, is independent of grain structure).

The effects of sand structure on volume changes may eventually happen when (and if) the locally stable grain cluster occurs which entraps some voids and resists the shearing. Usually, the grain skeleton is unstable during test allowing for rearrangements of "grain chains", especially at critical state where large deformations ("slow flow") occur. For instance, in Fig. 4 it is shown that in tests at elevated or high pressures (where crushing is pronounced) regular behavior complying with proposed Eq. (2) is obtained.

The collection of monodisperse spheres like glass beads, steel balls and even lead shot at low temperature will show similar behavior at lower pressures or shaking. When the larger pressures are applied and the strength of the loaded spheres is reached, the crushing and plastic deformation will occur and the monodispersity will be lost. By raising the pres- 
sures, it is expected that glass beads will continue to crush, similar to mineral sands, due to basically mineral constituents in glass. The metallic balls can also crush or deform further, but some differences can also be expected: due to local friction induced heat, the effects like melting or local welding can be expected, possibly leading to partial grain clustering.

Comment on $v d W$ model The continuum idealization from Fig. 3 presents a simple system (or model) consisting of two entities - one entity is incompressible containing all the matter in system, and the other is compressible but matterless (voids or free volume).

The pressure-volume relations for this system are described by original or adapted van der Waals equations Eqs. (1), (2). It is understood that the equation for real gases includes corrections on pressure and volume to comply with ideal gas pressure-volume behavior. The correction for volume of real gas means that only the free volume (or compressible entity) is taken into account. The correction for pressure (additional pressure) is material-dependent, acts on the whole volume and it is applied to corrected volume. In a model sense, the pressure correction can also be understood as an interaction parameter between two entities.

Real gases behave like ideal gases for volumes and temperatures well above critical. In these conditions, the volume of molecules compared to the total gas volume is at the level of impurity, and practically all the gas volume is free of matter.

This common observation with the reasoning based on former simple model leads to the conclusion that the universal ideal gas properties reflect the vacuum (or free space) properties and that possible (weak, non-bonded) interaction with matter is applied.

This is merely the possible deduction from the simple model, but, however, it is not the experimentally founded physical explanation.

It seems that more experimental and theoretical work with full scientific criticism is needed to distinguish the possible explanation of the presented empirical findings.

\section{Conclusion}

The pressure-volume behavior of mineral sands was investigated in terms of compressible fluid behavior. The results from previous [27] and this study show that the adapted van der Waals equation describes well the pressure-volume relation at critical state of mineral sands for a large range of applied pressures. More over, it is demonstrated that the ratio of appropriate empirical constants $A / C$ is close to 4 for all tests, and that the quantitative values of these constants are of the same order of magnitude as the corresponding constants of gases or other volatile fluids.
The constants $C$ and $A$ from Eq. (2) obtained experimentally from triaxial tests on mineral sands correlate well with ideal gas properties and molar properties of sands, and the basic proposed relations are in Eqs. (4) and (5).

These features demonstrate the similarity with empirical state equation of common compressible fluids. The basic difference is in ordinary thermal behavior and the proposed equation for mineral sands eventually represents the isothermal state in common coordinates.

If the mean pressure at critical state is understood to represent the intrinsic macroscopic properties of material, and if proven adequate for mineral sand descriptions, in the sense of continuum mechanics it may be used as an analogue for the spherical part of stress tensor [9-11,17-19]. It can also help in empirical evaluation of the internal energy changes in appropriate continuum theories (e.g like Granular Solid Hydrodynamics [59], with recent advances in [60]), or for calibration in numerical simulations, e.g. [61,62].

Since the presented findings came from tests on several mineral sands, more experimental work is needed to confirm the above relations for a wider range of pure substances or mineral mixtures, and to obtain the values of empirical coefficients to account for other non-assumed effects. Also, a lot of theoretical and experimental work is expected to give an insight (or underlying physics) into bulk empirical coefficients, considering the possible influences of forces and effects on microscale as well as possible indicated apparent attraction/repulsion effects on macroscale.

Open Access This article is distributed under the terms of the Creative Commons Attribution Noncommercial License which permits any noncommercial use, distribution, and reproduction in any medium, provided the original author(s) and source are credited.

\section{References}

1. Jaeger, H.M., Nagel, S.R.: Physics of the granular state. Science 255, 1523-1531 (1992)

2. Jaeger, H.M., Nagel, S.R., Behringer, R.P.: Granular solids, liquids, and gases. Rev. Mod. Phys. 68(4), 1259-1273 (1996)

3. Jaeger, H.M. : Chicago experiments on convection, compaction, and compression. In: Herrmann, H.J., Hovi, J.-P., Luding, S. (eds.) Physics of Dry Granular Media, pp. 553-584. Kluwer, Dordrecht (1998)

4. de Gennes, P.G.: Reflections on the mechanics of granular matter. Physica A 261, 267-293 (1998)

5. Jaeger, H.M., Shinbrot, T., Umbanhowar, P.B.: Does the granular matter? Proc. Natl. Acad. Sci. 97(24), 12959-12960 (2000)

6. Hinrichsen, H., Wolf, D.E. (eds.): The Physics of Granular Media. Wiley-VCH, Weinheim (2004)

7. Nedderman, R.M.: Statics and Kinematics of Granular Materials. Cambridge University Press, Cambridge (1992)

8. Antony, S.J., Hoyle, W., Ding, Y. (eds.): Granular Materials: Fundamentals and Applications. The Royal Society of Chemistry, Cambridge (2004)

9. Tardos, G.I.: A fluid mechanistic approach to slow, frictional flow of powders. Powder Technol. 92, 61-74 (1997) 
10. Tardos, G.I., McNamara, S., Talu, I.: Slow and intermediate flow of a frictional bulk powder in the Couette geometry. Powder Technol. 131, 23-39 (2003)

11. Massoudi, M., Phuoc, T.X.: Conduction and dissipation in the shearing flow of granular materials modeled as non-Newtonian fluids. Powder Technol. 175, 146-162 (2007)

12. Edwards, S.F., Grinev, D.V.: Statistical mechanics of granular materials: stress propagation and distribution of contact forces. Granul. Matter 4, 147 (2003)

13. Edwards, S.F., Oakeshott, R.B.S.: Theory of powders. Physica A 157, 1080 (1989)

14. Edwards, S.F.: Dynamics of complex flow. J. Stat. Phys. 62, 889 (1991)

15. Mehta, A., Edwards, S.F.: Statistical mechanics of powder mixtures. Physica A 157, 1091 (1989)

16. Henkes, S., Chakraborty, B.: Statistical mechanics framework for static granular matter. Phys. Rev. E 79, 061301 (2009)

17. Massoudi, M., Mehrabadi, M.M.: A continuum model for granular materials: considering dilatancy, and the Mohr-Coulomb criterion. Acta Mech. 152, 121-138 (2001)

18. Karner, S.L., Chester, F.M., Chester, J.S.: Towards a general state-variable constitutive relation to describe granular deformation. Earth Planet. Sci. Lett. 237, 940-950 (2005)

19. Humrickhouse, P.W., Sharpe, J.P., Corradini, M.L.: Comparison of hyperelastic models for granular materials. Phys. Rev. E 81, 011303 (2010)

20. Ciamarra, M.P., Coniglio, A., Nicodemi, M.: Thermodynamics and statistical mechanics of dense granular media. Phys. Rev. Lett. 97, 158001 (2006)

21. Herrmann, H.J.: On the thermodynamics of granular media. J. Phys. II France 3(4), 427-433 (1993)

22. Henkes, S., O'Hern, C.S., Chakraborty, B.: Entropy and temperature of a static granular assembly: an Ab Initio approach. Phys. Rev. Lett 99, 038002 (2007)

23. Makse, H.A., Kurchan, J.: Testing the thermodynamic approach to granular matter with a numerical model of a decisive experiment. Nature 415, 614-617 (2002)

24. Collins, I.F., Houlsby, G.T.: Application of thermomechanical principles to the modelling of geotechnical materials. Proc. Roy. Soc. Lond. A 453, 1975-2001 (1997)

25. Collins, I.F., Muhunthan, B.: On the relationship between stressdilatancy, anisotropy, and plastic dissipation for granular materials. Geotechnique 53(7), 611-618 (2003)

26. Collins, I.F.: Elastic/plastic models for soils and sands. Int. J. Mech. Sci. 47, 493-508 (2005)

27. Ivsic, T., Galovic, A., Kirin, D.: Sand as a compressible fluid. Physica A 277, 47-61 (2000)

28. Sitharam, T.G., Vinod, J.S.: Critical state behaviour of granular materials from isotropic and rebounded paths: DEM simulations. Granul. Matter 11, 33-42 (2009)

29. Luding, S., Alonso-Maroquin, F.: The critical-state yielding stress (termination locus) of adhesive powders from a single numerical experiment. Granul. Matter 13, 109-119 (2011)

30. Schofield, A., Wroth, P.: Critical State Soil Mechanics. McGrawHill, London (1968)

31. Wood, D.M.: Soil Behaviour and Critical State Soil Mechanics. Cambridge University Press, Cambridge (1990)

32. Atkinson, J.: An Introduction to the Mechanics of Soils and Foundations. McGraw-Hill Book Company, London (1993)

33. Poorooshasb, H.B.: Description of flow of sand using state parameters. Comput. Geotech. 8, 195-218 (1989)

34. Brujic, J., Edwards, S.F., Grinev, D.: Jammed systems in slow flow need a new statistical mechanics. Phil. Trans. R. Soc. Lond. A 361, 741-751 (2003)
35. Potiguar, F.Q., Makse, H.A.: Effective temperature and jamming transition in dense, gently sheared granular assemblies. Eur. Phys. J. E, 19, 171 (2006)

36. Majmudar, T.S., Sperl, M., Luding, S., Behringer, R.P.: The jamming transition in granular systems. Phys. Rev. Lett. 98, 058001 (2007)

37. Silbert, L.E., Silbert, M.: Long-wavelength structural anomalies in jammed systems. Phys. Rev. E 80, 041304 (2009)

38. Otsuki, M., Hayakawa, H.: Critical behaviors of sheared frictionless granular materials near the jamming transition. Phys. Rev. E 80, 011308 (2009)

39. Campbell, C.S.: Granular shear flow at the elastic limit. J.Fluid Mech. 465, 261-291 (2002)

40. Zhu, H., Mehrabadi, M.M., Massoudi, M.: The frictional flow of a dense granular material based on the dilatant double shearing model. Comput. Math. Appl. 53, 244-259 (2007)

41. Reynolds, O.: On the dilatancy of media composed of rigid particles in contact with experimental illustrations. Phil. Mag. S. 520, 469481 (1885)

42. Reynolds, O.: Experiments showing dilatancy. A property of granular material, possibly connected with gravitation. Proc. Royal. Inst. Great B. 11, 354-363 (1886)

43. Bardet, J.P.: Experimental Soil Mechanics. Prentice Hall, New Jersey (1997)

44. Jefferies, M., Been, K.: Soil Liquefaction: A Critical State Approach. Taylor and Francis, New York (2006)

45. Fioravante, V., Capoferri, R.: Automatic volume measuring device for testing dry soils : Martina. ASTM Geotech. Test. J. 20(4), 423432 (1997)

46. Vesić, A.S., Clough, G.W.: Behavior of granular materials under high stresses. J. Soil Mech. Found. Eng. Div. ASCE 94(SM3), 661688 (1968)

47. Lo, K.Y., Roy, M.: Response of particulate materials at high pressures. Soils Found. 13(1), 1-14 (1973)

48. Yamamuro, J.A., Lade, P.V.: Drained sand behavior in axisymmetric tests at high pressures. J. Geotech. Eng. ASCE 122(2), 109119 (1996)

49. Yamamuro, J.A., Bopp, P.A., Lade, P.V.: One-dimensional compression of sands at high pressures. J. Geotech. Eng. ASCE 122(2), 147-154 (1996)

50. Chu, J.: An experimental examination of the critical state and other similar concepts for granular soils. Can. Geotech. J. 32, 10651075 (1995)

51. Butterfield, R.: A natural compression law for soils (an advance on e - $\log$ p'). Geotechnique 29 (4) 469-480 (1979); includes interpreted tests from: Parry, R.H.G.: Triaxial compression and extension tests on remoulded saturated clay. Geotechnique 10 (4) 166-180 (1960)

52. Yu, H.S., Khong, C.D., Wang, J., Zhang, G.: Experimental evaluation and extension of a simple critical state model for sand. Granul. Matter 7, 213-225 (2005)

53. Schalley, C.A : ch.1.2 The noncovalent bond: a brief overview. In: Analytical Methods in Supramolecular Chemistry, WILEY-VCH, Weinheim (2007)

54. Yu, A.B., Feng, C.L., Zou, R.P., Yang, R.Y.: On the relationship between porosity and interparticle forces. Powder Technol. 130, 70-76 (2003)

55. Wang, Y.H., Siu, W.K.: Structure characteristics and mechanical properties of kaolinite soils. I. Surface charges and structural characterizations. Can. Geotech. J. 43, 587-600 (2006)

56. Hardin, B.O: Crushing of soil particles. J.Geotech. Eng. ASCE 111(10), 1177-1192 (1985)

57. Lade, P.V., Yamamuro, J.A., Bopp, P.A.: Significance of particle crushing in granular materials. J. Geotech. Eng. ASCE 122(4), 309316 (1996) 
58. Arslan, H., Baykal, G., Sture, S.: Analysis of the influence of crushing on the behavior of granular materials under shear. Granul. Matter 11, 87-97 (2009)

59. Jiang, Y., Liu, M.: Granular solid hydrodynamics. Granul. Matter 11, 139-156 (2009)

60. Gudehus, G., Jiang, Y., Liu, M.: Seismo- and thermodynamics of granular solids. Granul. Matter 13, 319-340 (2011)
61. Luding, S.: A global equation of state of two-dimensional hard sphere systems. Phys. Rev. E 63, 042201 (2001)

62. Welker, P.R., McNamara, S.C.: What triggers failure in frictional granular assemblies? Phys. Rev. E 79, 061305 (2009) 